\title{
Ocean Shipping Container Availability Report
}

A weekly publication of the Agricultural Marketing Service

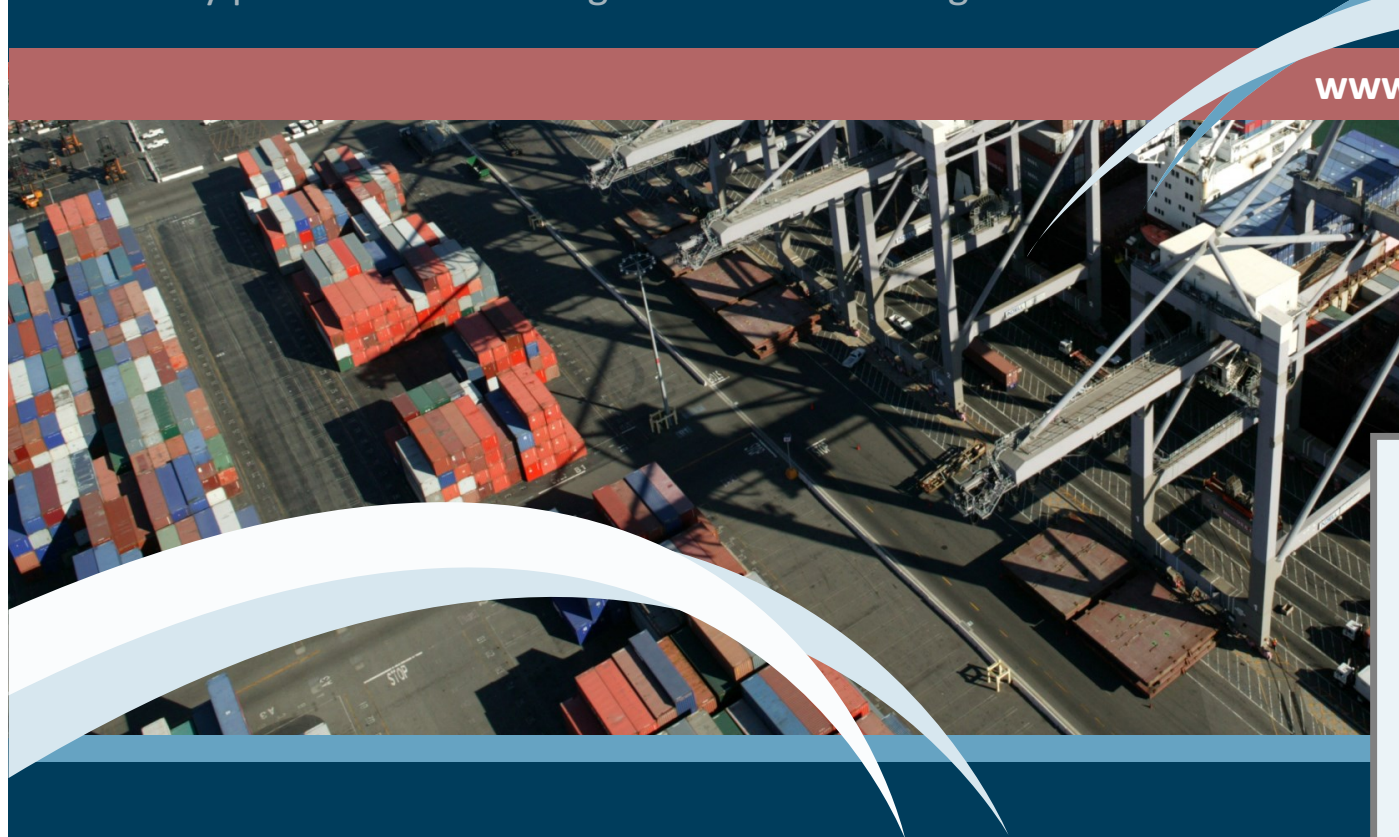

The Ocean Shipping Container Availability Report (OSCAR) provides a weekly snapshot of the availability of marine shipping containers at 18 different intermodal locations for the westbound transpacific trade lanes. The data shows the availability for five types of equipment, including 20 and $40 \mathrm{ft}$ dry, 20 and $40 \mathrm{ft}$ refrigerated, and 40ft high-cube containers, and provides estimates of equipment availability for the current week and projections two weeks out. The data are voluntarily provided to AMS by six members of the Transpacific Stabilization Agreement (TSA).

Container Availability by Location:

National Overview Maps P. 2-4

Container Availability by Location:

Charleston, SC P.5

Chicago, IL P.6

Cincinnati, OH P.7

Columbus, $\mathrm{OH}$ P.8

Dallas, TX P.9

Denver, CO P.10

Houston, TX P.11

Kansas City, MO P.12

Los Angeles and Long Beach, CA P.13

Memphis, TN P.14 Minneapolis, MN P.15

New Orleans, LA P.16

New York, NY P.17

Norfolk, VA P.18

Oakland, CA P.19

Savannah, GA P.20

Seattle, WA P.21

Tacoma, WA P.22

Understanding this Report P.23

To subscribe to OSCAR, e-mail: April.Taylor@ams.usda.gov 


\section{National Overview}

\section{Week of April 29-May 5, 2015}

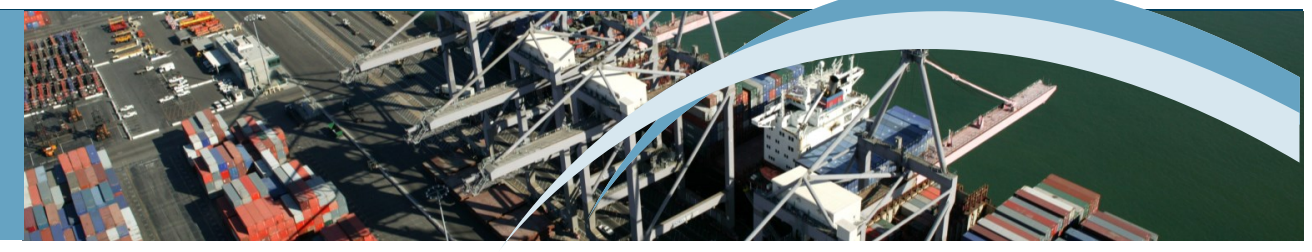

Six-Month Snapshot of Average Weekly Throughput

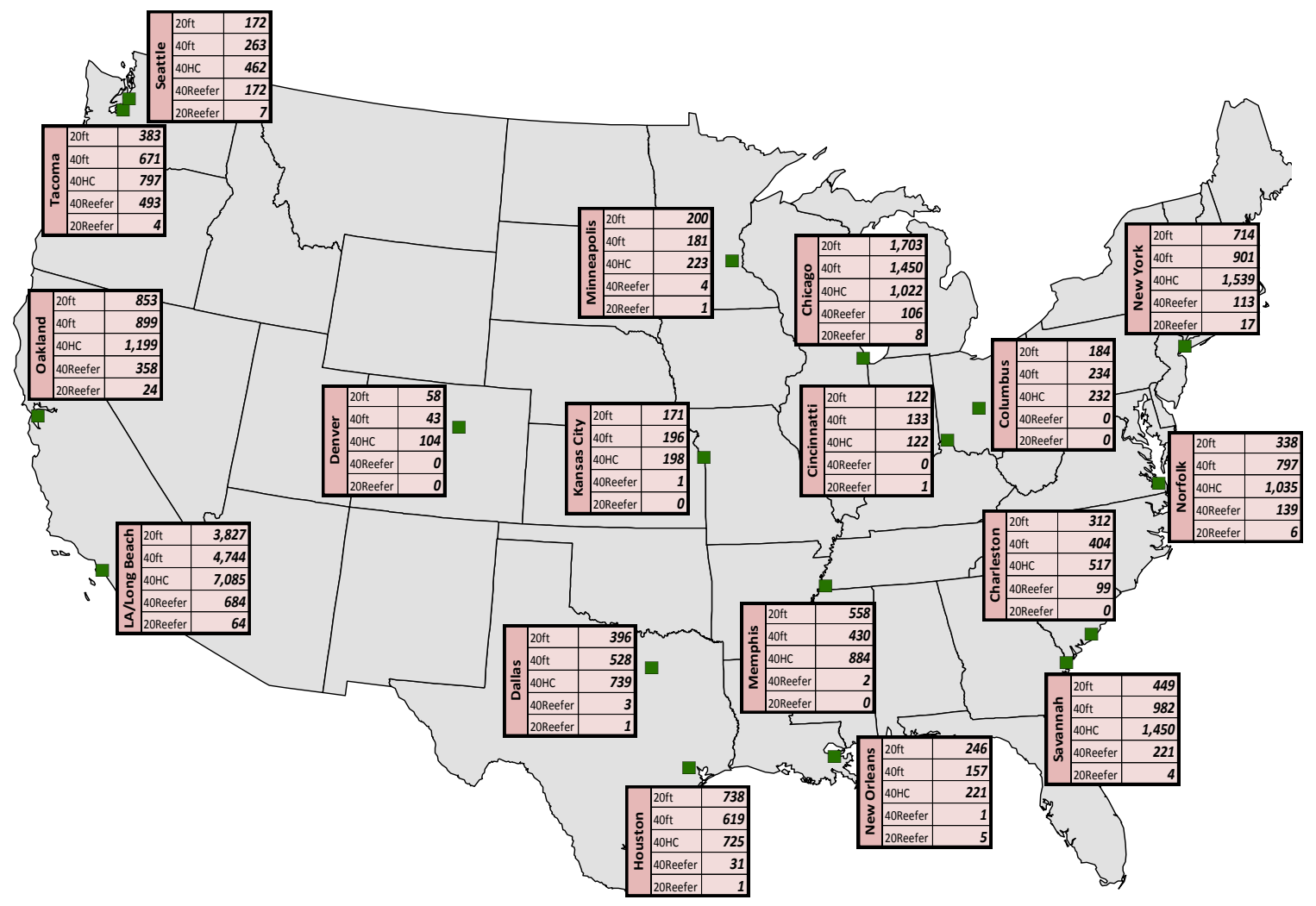

Average Weekly Availability for 20ft Dry Containers

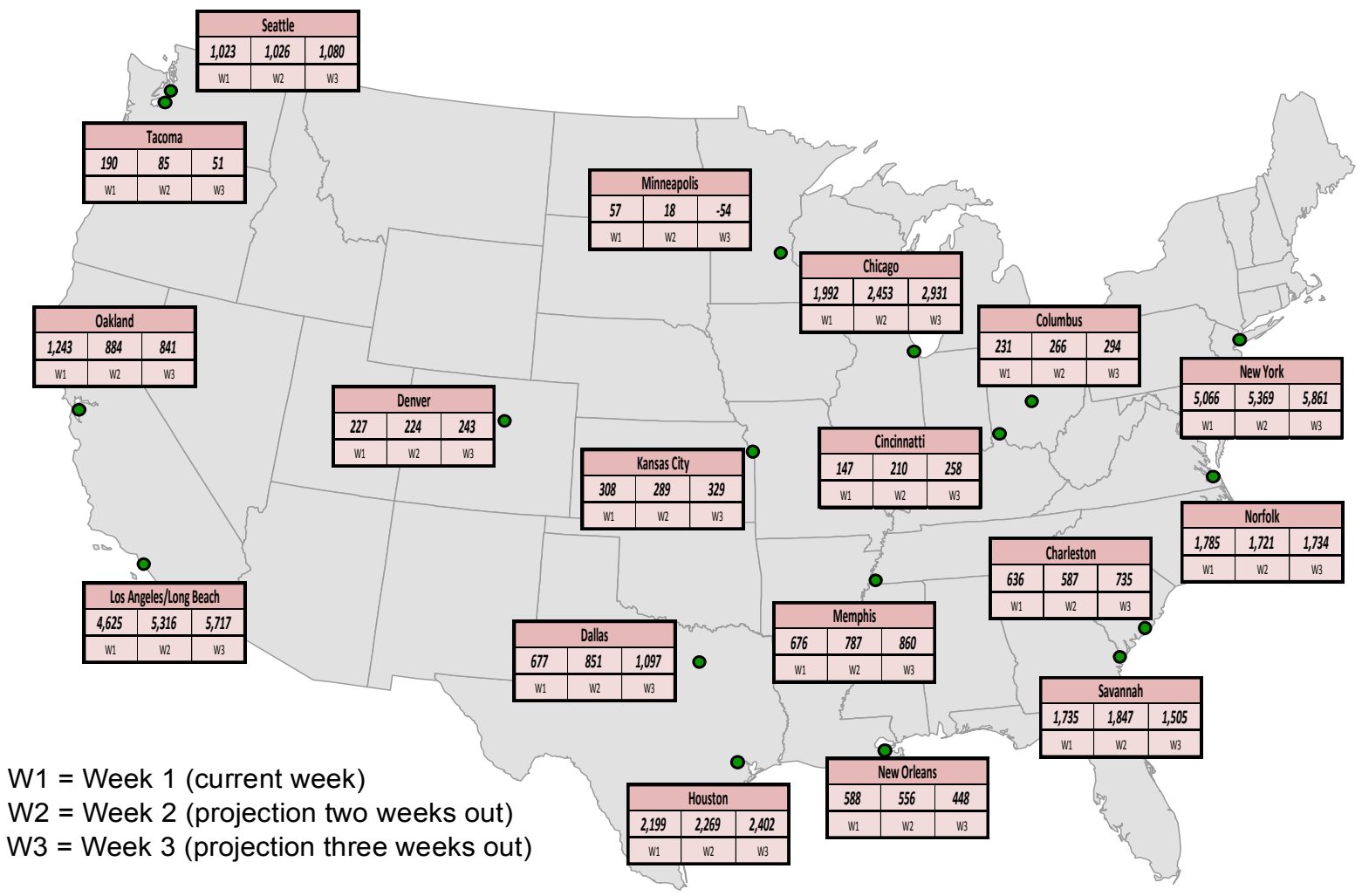




\section{National Overview}

\section{Week of April 29-May 5, 2015}

\section{Average Weekly Availability for 40ft Dry Containers}

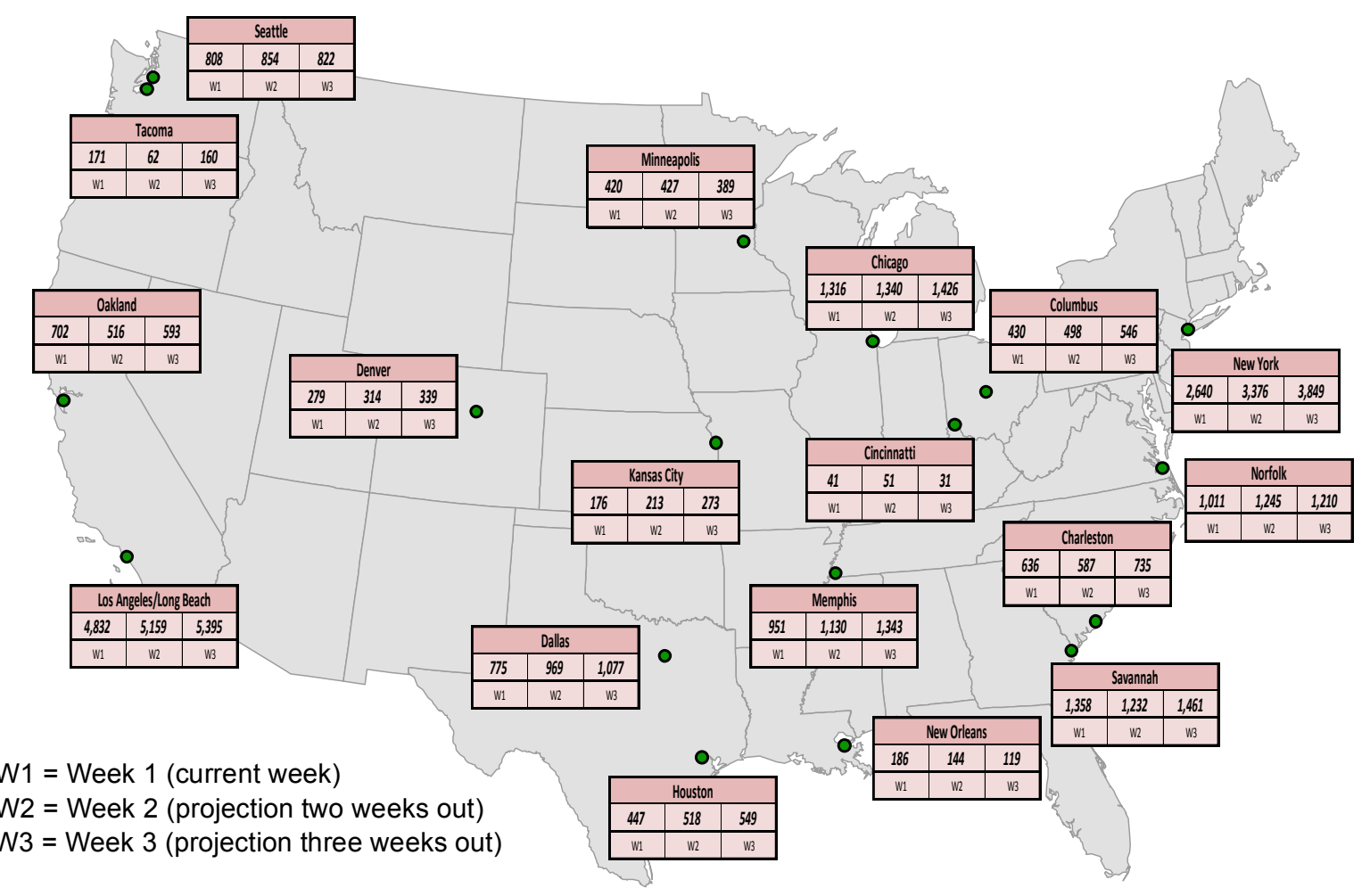

\section{Average Weekly Availability for 40ft High Cube Containers}

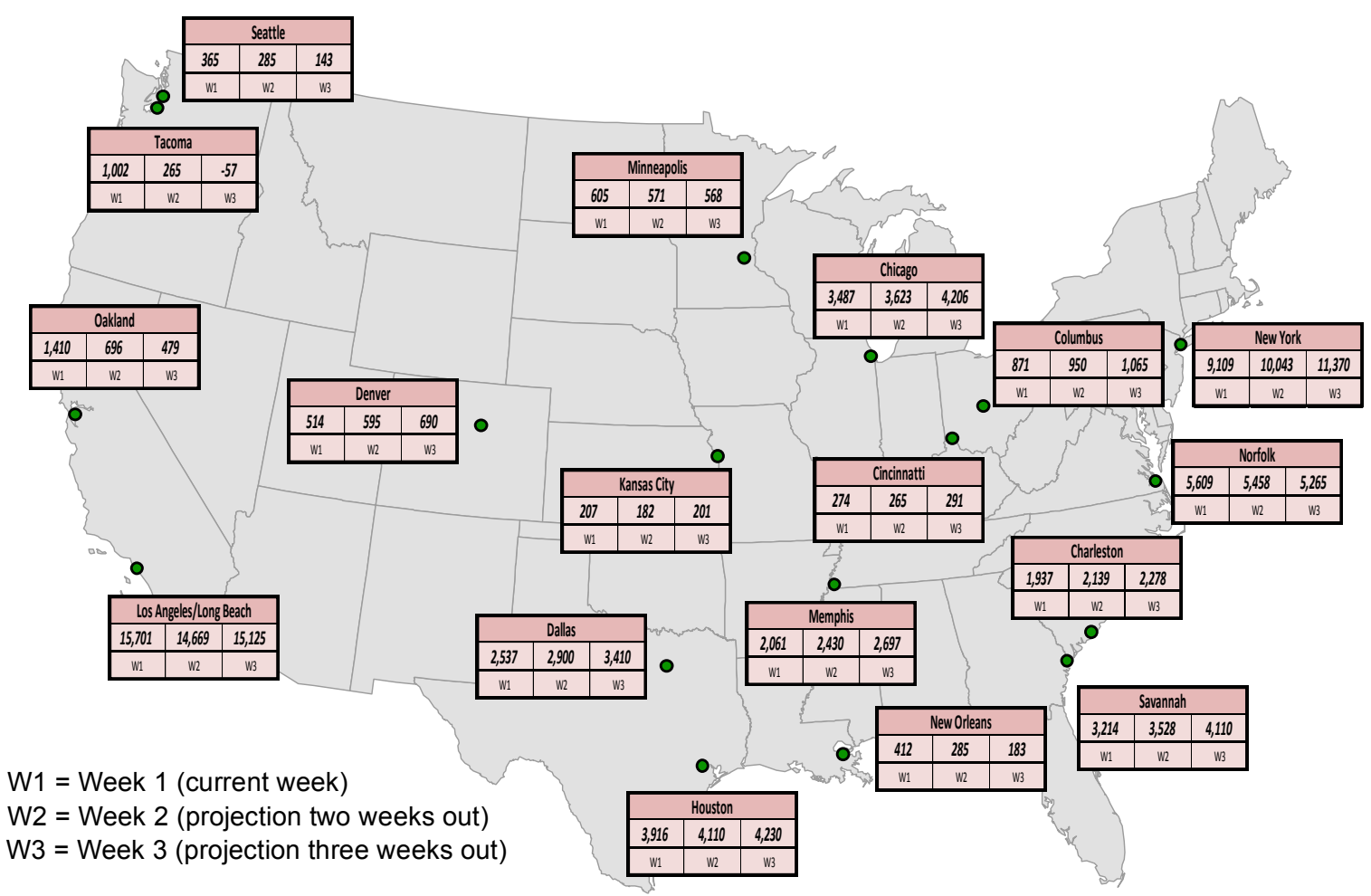




\section{National Overview}

\section{Week of April 29-May 5, 2015}

\section{Average Weekly Availability for 40ft Refrigerated Containers}

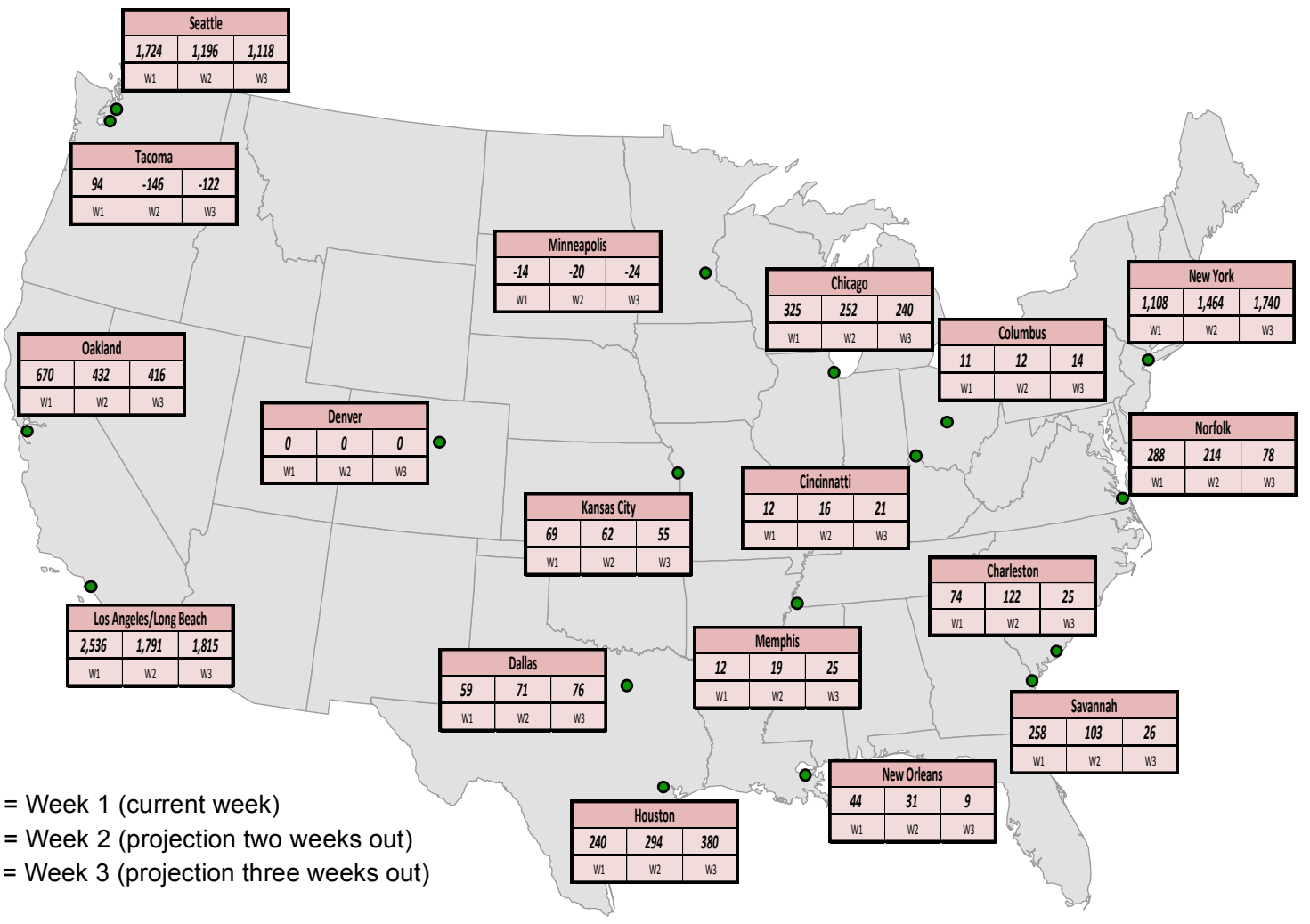

\section{Average Weekly Availability for 20ft Refrigerated Containers}

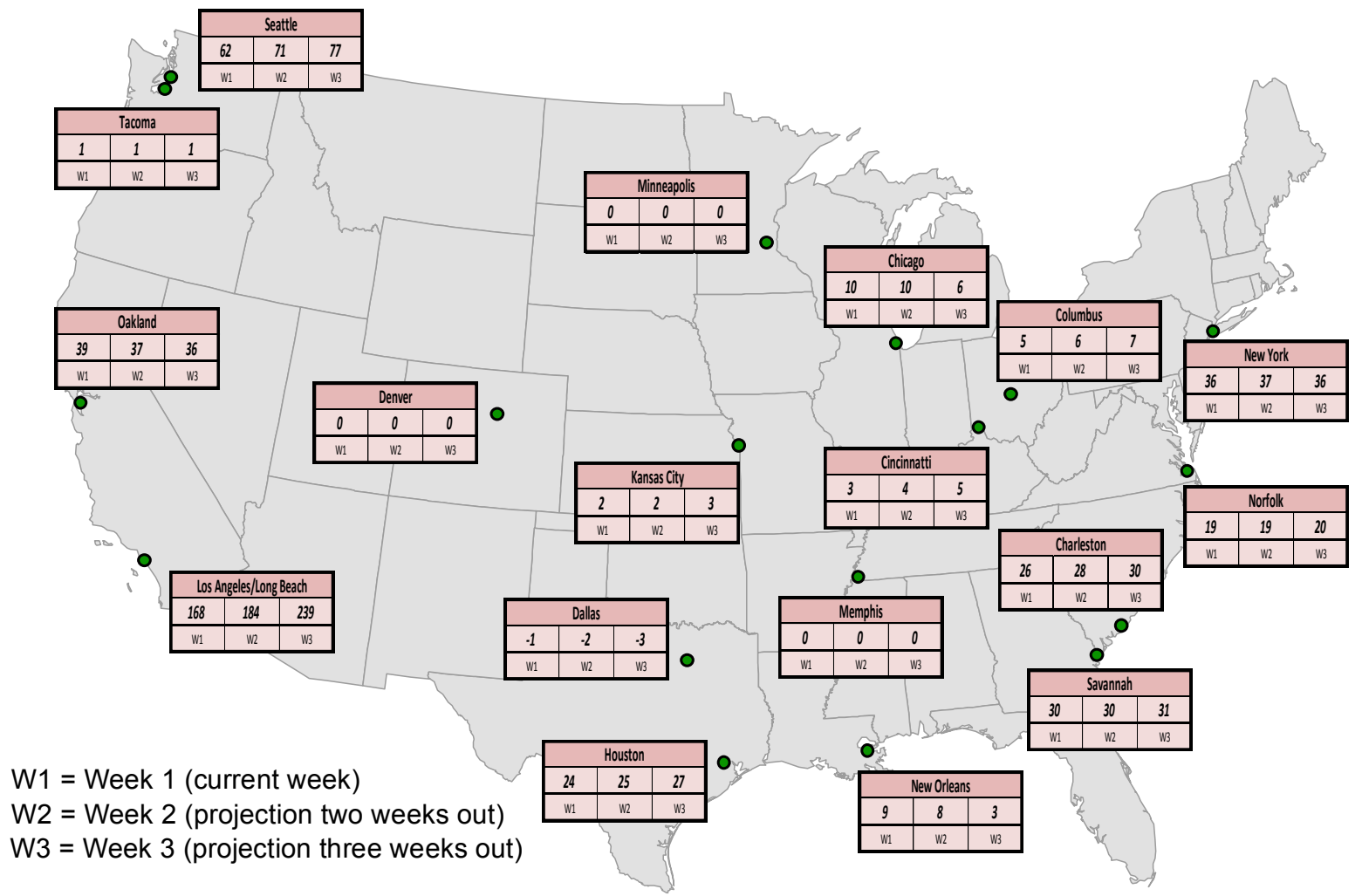




\section{Charleston, SC}

\begin{tabular}{|c|c|c|c|c|c|c|c|}
\hline \multicolumn{8}{|c|}{ Charleston } \\
\hline \multicolumn{8}{|c|}{ 20ft Dry } \\
\hline & \multicolumn{6}{|c|}{ Carrier Availability Responses } & Total \\
\hline Current Week: Apr 29-May 5 & 261 & 131 & 101 & 71 & 48 & 24 & 636 \\
\hline Week 2: May 6-12 & 175 & 157 & 101 & 62 & 62 & 30 & 587 \\
\hline Week 3: May 13-19 & 290 & 186 & 101 & 58 & 50 & 50 & 735 \\
\hline \multicolumn{8}{|c|}{ 40ft Dry } \\
\hline & \multicolumn{6}{|c|}{ Carrier Availability Responses } & Total \\
\hline Current Week: Apr 29-May 5 & 359 & 316 & 122 & 118 & 29 & 25 & 969 \\
\hline Week 2: May 6-12 & 423 & 316 & 90 & 127 & 50 & 72 & 1,078 \\
\hline Week 3: May 13-19 & 486 & 316 & 132 & 139 & 50 & 40 & 1,163 \\
\hline \multicolumn{8}{|c|}{ 40ft High Cube } \\
\hline & \multicolumn{6}{|c|}{ Carrier Availability Category Responses } & Total \\
\hline Current Week: Apr 29-May 5 & 807 & 584 & 276 & 181 & 47 & 42 & 1,937 \\
\hline Week 2: May 6-12 & 965 & 584 & 303 & 198 & 50 & 39 & 2,139 \\
\hline Week 3: May 13-19 & 1,122 & 584 & 340 & 167 & 30 & 35 & 2,278 \\
\hline \multicolumn{8}{|c|}{ 40ft Refrigerated } \\
\hline & \multicolumn{6}{|c|}{ Carrier Availability Category Responses } & Total \\
\hline Current Week: Apr 29-May 5 & 130 & 9 & 8 & -2 & -71 & . & 74 \\
\hline Week 2: May 6-12 & 117 & 9 & 10 & -2 & -12 & . & 122 \\
\hline Week 3: May 13-19 & 106 & 7 & 15 & -1 & -102 & . & 25 \\
\hline \multicolumn{8}{|c|}{ 20ft Refrigerated } \\
\hline & \multicolumn{6}{|c|}{ Carrier Availability Category Responses } & Total \\
\hline Current Week: Apr 29-May 5 & 24 & 2 & . & . & . & . & 26 \\
\hline Week 2: May 6-12 & 27 & 1 & . & . & . & . & 28 \\
\hline Week 3: May 13-19 & 30 & 0 & . & . & . & . & 30 \\
\hline
\end{tabular}

Note: "." denotes no response from carriers.

\begin{tabular}{|l|c|c|}
\hline \multicolumn{3}{|c|}{ Six-Month Snapshot of Average Weekly } \\
Throughput \\
June 2012-November 2012 \\
\hline Container Types & $\begin{array}{c}\text { Average Total Weekly } \\
\text { Throughput* }\end{array}$ & $\begin{array}{c}\text { Range of Individual } \\
\text { Carrier Responses }\end{array}$ \\
\hline $\mathbf{2 0 f t}$ & 312 & $0 \ldots 108$ \\
$\mathbf{4 0 f t}$ & 404 & $0 \ldots 200$ \\
$\mathbf{4 0 f t}$ High Cubes & 517 & $17 . . .200$ \\
40ft Refrigerated & 99 & $0 . .60$ \\
20ft Refrigerated & 0 & $0 . . .0$ \\
\hline
\end{tabular}

*Data reflect the sum of the individual carriers' average weekly throughput over a 6-month timeframe 


\section{Chicago, IL}

\begin{tabular}{|c|c|c|c|c|c|c|c|}
\hline \multicolumn{8}{|c|}{ Chicago } \\
\hline \multicolumn{8}{|c|}{ 20ft Dry } \\
\hline & \multicolumn{6}{|c|}{ Carrier Availability Category Responses } & Total \\
\hline Current Week: Apr 29-May 5 & 604 & 418 & 408 & 401 & 147 & 14 & 1,992 \\
\hline Week 2: May 6-12 & 913 & 300 & 408 & 742 & 70 & 20 & 2,453 \\
\hline Week 3: May 13-19 & 1,210 & 200 & 408 & 1,207 & -112 & 18 & 2,931 \\
\hline \multicolumn{8}{|c|}{ 40ft Dry } \\
\hline & \multicolumn{6}{|c|}{ Carrier Availability Category Responses } & Total \\
\hline Current Week: Apr 29-May 5 & 447 & 426 & 205 & 148 & 85 & 5 & 1,316 \\
\hline Week 2: May 6-12 & 390 & 541 & 180 & 148 & -69 & 150 & 1,340 \\
\hline Week 3: May 13-19 & 369 & 657 & 173 & 148 & -121 & 200 & 1,426 \\
\hline \multicolumn{8}{|c|}{ 40ft High Cube } \\
\hline & \multicolumn{6}{|c|}{ Carrier Availability Category Responses } & Total \\
\hline Current Week: Apr 29-May 5 & 1,052 & 854 & 697 & 513 & 229 & 142 & 3,487 \\
\hline Week 2: May 6-12 & 1,052 & 1,006 & 968 & 403 & 150 & 44 & 3,623 \\
\hline Week 3: May 13-19 & 1,052 & 1,177 & 1,141 & 500 & 200 & 136 & 4,206 \\
\hline \multicolumn{8}{|c|}{ 40ft Refrigerated } \\
\hline & \multicolumn{6}{|c|}{ Carrier Availability Category Responses } & Total \\
\hline Current Week: Apr 29-May 5 & 144 & 115 & 66 & 0 & 0 & . & 325 \\
\hline Week 2: May 6-12 & 137 & 105 & 10 & 0 & 0 & . & 252 \\
\hline Week 3: May 13-19 & 117 & 93 & 30 & 0 & 0 & . & 240 \\
\hline \multicolumn{8}{|c|}{ 20ft Refrigerated } \\
\hline & \multicolumn{6}{|c|}{ Carrier Availability Category Responses } & Total \\
\hline Current Week: Apr 29-May 5 & 5 & 5 & . & . & . & . & 10 \\
\hline Week 2: May 6-12 & 8 & 2 & . & . & . & . & 10 \\
\hline Week 3: May 13-19 & 7 & -1 & . & . & . & . & 6 \\
\hline
\end{tabular}

Note: "." denotes no response from carriers.

\begin{tabular}{|l|c|c|}
\hline \multicolumn{3}{|c|}{ Six-Month Snapshot of Average Weekly } \\
Throughput \\
June 2012-November $\mathbf{2 0 1 2}$ \\
\hline Container Types & $\begin{array}{c}\text { Average Total Weekly } \\
\text { Throughput* }\end{array}$ & $\begin{array}{c}\text { Range of Individual } \\
\text { Carrier Responses }\end{array}$ \\
\hline $\mathbf{2 0 f t}$ & 1,703 & $93 . .600$ \\
$\mathbf{4 0 f t}$ & 1,450 & $5 \ldots 302$ \\
$\mathbf{4 0 f t}$ High Cubes & 1,022 & $34 \ldots 405$ \\
$\mathbf{4 0 f t}$ Refrigerated & 106 & $0 . . .51$ \\
20ft Refrigerated & 8 & $0 . . .4$ \\
\hline
\end{tabular}

* Data reflect the sum of the individual carriers' average weekly throughput over a 6-month timeframe 


\section{Cincinnati, $\mathrm{OH}$}

\section{Cincinnatti}

20ft Dry

\begin{tabular}{|c|c|c|c|c|c|c|c|}
\hline & \multicolumn{6}{|c|}{ Carrier Availability Category Responses } & \multirow{2}{*}{$\begin{array}{c}\text { Total } \\
147\end{array}$} \\
\hline Current Week: Apr 29-May 5 & 74 & 41 & 24 & 17 & -4 & -5 & \\
\hline Week 2: May 6-12 & 99 & 41 & 30 & 17 & 3 & 20 & 210 \\
\hline Week 3: May 13-19 & 130 & 50 & 38 & 17 & 3 & 20 & 258 \\
\hline \multicolumn{8}{|c|}{ 40ft Dry } \\
\hline & \multicolumn{6}{|c|}{ Carrier Availability Category Responses } & Total \\
\hline Current Week: Apr 29-May 5 & 19 & 14 & 13 & 8 & 3 & -16 & 41 \\
\hline Week 2: May 6-12 & -2 & 10 & 9 & 6 & 44 & -16 & 51 \\
\hline Week 3: May 13-19 & -12 & 10 & 5 & 5 & 39 & -16 & 31 \\
\hline \multicolumn{8}{|c|}{ 40ft High Cube } \\
\hline & \multicolumn{6}{|c|}{ Carrier Availability Category Responses } & Total \\
\hline Current Week: Apr 29-May 5 & 164 & 59 & 28 & 13 & 6 & 4 & 274 \\
\hline Week 2: May 6-12 & 164 & 60 & 10 & 11 & 18 & 2 & 265 \\
\hline Week 3: May 13-19 & 164 & 74 & 10 & 12 & 31 & 0 & 291 \\
\hline \multicolumn{8}{|c|}{ 40ft Refrigerated } \\
\hline & \multicolumn{6}{|c|}{ Carrier Availability Category Responses } & Total \\
\hline Current Week: Apr 29-May 5 & 12 & 0 & . & . & . & . & 12 \\
\hline Week 2: May 6-12 & 16 & 0 & . & . & . & . & 16 \\
\hline Week 3: May 13-19 & 21 & 0 & . & . & . & . & 21 \\
\hline \multicolumn{8}{|c|}{ 20ft Refrigerated } \\
\hline & \multicolumn{6}{|c|}{ Carrier Availability Category Responses } & Total \\
\hline Current Week: Apr 29-May 5 & 3 & . & . & . & . & . & 3 \\
\hline Week 2: May 6-12 & 4 & . & . & . & . & . & 4 \\
\hline Week 3: May 13-19 & 5 & . & . & . & . & . & 5 \\
\hline
\end{tabular}

Note: "." denotes no response from carriers.

\begin{tabular}{|l|c|c|}
\hline \multicolumn{3}{|c|}{ Six-Month Snapshot of Average Weekly } \\
Throughput \\
June 2012-November 2012 \\
\hline Container Types & $\begin{array}{c}\text { Average Total Weekly } \\
\text { Throughput* }\end{array}$ & $\begin{array}{c}\text { Range of Individual } \\
\text { Carrier Responses }\end{array}$ \\
\hline $\mathbf{2 0 f t}$ & 122 & $7 \ldots 35$ \\
$\mathbf{4 0 f t}$ & 133 & $8 \ldots 30$ \\
40ft High Cubes & 122 & $11 \ldots 35$ \\
40ft Refrigerated & 0 & $0 \ldots 0$ \\
20ft Refrigerated & 1 & $0 \ldots 1$ \\
\hline
\end{tabular}

*Data reflect the sum of the individual carriers' average weekly throughput over a 6-month timeframe 


\section{Columbus, $\mathrm{OH}$}

\begin{tabular}{|c|c|c|c|c|c|c|c|}
\hline \multicolumn{8}{|c|}{ Columbus } \\
\hline \multicolumn{8}{|c|}{ 20ft Dry } \\
\hline & \multicolumn{6}{|c|}{ Carrier Availability Category Responses } & Total \\
\hline Current Week: Apr 29-May 5 & 88 & 55 & 54 & 39 & -1 & -4 & 231 \\
\hline Week 2: May 6-12 & 108 & 94 & 54 & 30 & -2 & -18 & 266 \\
\hline Week 3: May 13-19 & 134 & 109 & 54 & 30 & 0 & -33 & 294 \\
\hline \multicolumn{8}{|c|}{ 40ft Dry } \\
\hline & \multicolumn{6}{|c|}{ Carrier Availability Category Responses } & Total \\
\hline Current Week: Apr 29-May 5 & 142 & 128 & 127 & 50 & 1 & -18 & 430 \\
\hline Week 2: May 6-12 & 142 & 158 & 135 & 63 & 0 & 0 & 498 \\
\hline Week 3: May 13-19 & 142 & 184 & 155 & 65 & 0 & 0 & 546 \\
\hline \multicolumn{8}{|c|}{ 40ft High Cube } \\
\hline & \multicolumn{6}{|c|}{ Carrier Availability Category Responses } & Total \\
\hline Current Week: Apr 29-May 5 & 436 & 250 & 79 & 75 & 46 & -15 & 871 \\
\hline Week 2: May 6-12 & 436 & 329 & 40 & 90 & 58 & -3 & 950 \\
\hline Week 3: May 13-19 & 436 & 421 & 40 & 101 & 74 & -7 & 1,065 \\
\hline \multicolumn{8}{|c|}{ 40ft Refrigerated } \\
\hline & \multicolumn{6}{|c|}{ Carrier Availability Category Responses } & Total \\
\hline Current Week: Apr 29-May 5 & 11 & 0 &. & . & . & . & 11 \\
\hline Week 2: May 6-12 & 12 & 0 & . & . & . & . & 12 \\
\hline Week 3: May 13-19 & 14 & 0 &. & . & . & . & 14 \\
\hline \multicolumn{8}{|c|}{ 20ft Refrigerated } \\
\hline & \multicolumn{6}{|c|}{ Carrier Availability Category Responses } & Total \\
\hline Current Week: Apr 29-May 5 & 5 & . &. & . & . & . & 5 \\
\hline Week 2: May 6-12 & 6 & . & . & . & . & . & 6 \\
\hline Week 3: May 13-19 & 7 & . & . & . & . & . & 7 \\
\hline
\end{tabular}

Note: "." denotes no response from carriers.

\begin{tabular}{|l|c|c|}
\hline \multicolumn{3}{|c|}{ Six-Month Snapshot of Average Weekly } \\
Throughput \\
June 2012-November 2012 \\
\hline \multirow{2}{*}{ Container Types } & $\begin{array}{c}\text { Average Total Weekly } \\
\text { Throughput* }\end{array}$ & $\begin{array}{c}\text { Range of Individual } \\
\text { Carrier Responses }\end{array}$ \\
\hline $\mathbf{2 0 f t}$ & 184 & $8 \ldots 60$ \\
$\mathbf{4 0 f t}$ & 234 & $6 \ldots 80$ \\
$\mathbf{4 0 f t ~ H i g h ~ C u b e s ~}$ & 232 & $15 \ldots 60$ \\
$\mathbf{4 0 f t}$ Refrigerated & 0 & $0 \ldots 0$ \\
$\mathbf{2 0 f t}$ Refrigerated & 0 & $0 \ldots 0$ \\
\hline
\end{tabular}

*Data reflect the sum of the individual carriers' average weekly throughput over a 6-month timeframe 


\section{Dallas, TX}

\begin{tabular}{|c|c|c|c|c|c|c|c|}
\hline \multicolumn{8}{|c|}{ Dallas } \\
\hline \multicolumn{8}{|c|}{ 20ft Dry } \\
\hline & \multicolumn{6}{|c|}{ Carrier Availability Category Responses } & Total \\
\hline Current Week: Apr 29-May 5 & 235 & 178 & 89 & 72 & 69 & 34 & 677 \\
\hline Week 2: May 6-12 & 296 & 226 & 90 & 155 & 50 & 34 & 851 \\
\hline Week 3: May 13-19 & 383 & 284 & 100 & 246 & 50 & 34 & 1,097 \\
\hline \multicolumn{8}{|c|}{ 40ft Dry } \\
\hline & \multicolumn{6}{|c|}{ Carrier Availability Category Responses } & Total \\
\hline Current Week: Apr 29-May 5 & 159 & 158 & 157 & 147 & 94 & 60 & 775 \\
\hline Week 2: May 6-12 & 240 & 106 & 310 & 153 & 100 & 60 & 969 \\
\hline Week 3: May 13-19 & 296 & 42 & 449 & 130 & 100 & 60 & 1,077 \\
\hline \multicolumn{8}{|c|}{ 40ft High Cube } \\
\hline & \multicolumn{6}{|c|}{ Carrier Availability Category Responses } & Total \\
\hline Current Week: Apr 29-May 5 & 813 & 753 & 393 & 384 & 117 & 77 & 2,537 \\
\hline Week 2: May 6-12 & 1,045 & 559 & 508 & 384 & 254 & 150 & 2,900 \\
\hline Week 3: May 13-19 & 1,301 & 525 & 652 & 384 & 398 & 150 & 3,410 \\
\hline \multicolumn{8}{|c|}{ 40ft Refrigerated } \\
\hline & \multicolumn{6}{|c|}{ Carrier Availability Category Responses } & Total \\
\hline Current Week: Apr 29-May 5 & 38 & 21 & 0 & . & . & . & 59 \\
\hline Week 2: May 6-12 & 46 & 25 & 0 & . & . & . & 71 \\
\hline Week 3: May 13-19 & 49 & 27 & 0 & . & . & . & 76 \\
\hline \multicolumn{8}{|c|}{ 20ft Refrigerated } \\
\hline & \multicolumn{6}{|c|}{ Carrier Availability Category Responses } & Total \\
\hline Current Week: Apr 29-May 5 & -1 & . & . & . & . & . & -1 \\
\hline Week 2: May 6-12 & -2 & . & . & . & . & . & -2 \\
\hline Week 3: May 13-19 & -3 & . & . & . & . & . & -3 \\
\hline
\end{tabular}

Note: "." denotes no response from carriers.

\begin{tabular}{|l|c|c|}
\hline \multicolumn{3}{|c|}{ Six-Month Snapshot of Average Weekly } \\
Throughput \\
June 2012-November 2012 \\
\hline \multirow{2}{*}{ Container Types } & $\begin{array}{c}\text { Average Total Weekly } \\
\text { Throughput* }\end{array}$ & $\begin{array}{c}\text { Range of Individual } \\
\text { Carrier Responses }\end{array}$ \\
\hline $\mathbf{2 0 f t}$ & 396 & $5 \ldots 150$ \\
$\mathbf{4 0 f t}$ & 528 & $12 \ldots .250$ \\
$\mathbf{4 0 f t}$ High Cubes & 739 & $22 \ldots .250$ \\
$\mathbf{4 0 f t}$ Refrigerated & 3 & $0 \ldots 3$ \\
$\mathbf{2 0 f t}$ Refrigerated & 1 & $0 . . .1$ \\
\hline
\end{tabular}

*Data reflect the sum of the individual carriers' average weekly throughput over a 6-month timeframe 


\section{Denver, CO}

\begin{tabular}{|c|c|c|c|c|c|c|c|}
\hline \multicolumn{8}{|c|}{ Denver } \\
\hline \multicolumn{8}{|c|}{ 20ft Dry } \\
\hline & \multicolumn{6}{|c|}{ Carrier Availability Category Responses } & Total \\
\hline Current Week: Apr 29-May 5 & 91 & 55 & 38 & 28 & 16 & -1 & 227 \\
\hline Week 2: May 6-12 & 106 & 55 & 43 & 10 & 11 & -1 & 224 \\
\hline Week 3: May 13-19 & 122 & 55 & 48 & 10 & 9 & -1 & 243 \\
\hline \multicolumn{8}{|c|}{ 40ft Dry } \\
\hline & \multicolumn{6}{|c|}{ Carrier Availability Category Responses } & Total \\
\hline Current Week: Apr 29-May 5 & 176 & 54 & 23 & 13 & 12 & 1 & 279 \\
\hline Week 2: May 6-12 & 203 & 54 & 34 & 10 & 10 & 3 & 314 \\
\hline Week 3: May 13-19 & 231 & 54 & 39 & 5 & 10 & 0 & 339 \\
\hline \multicolumn{8}{|c|}{ 40ft High Cube } \\
\hline & \multicolumn{6}{|c|}{ Carrier Availability Category Responses } & Total \\
\hline Current Week: Apr 29-May 5 & 158 & 150 & 112 & 72 & 17 & 5 & 514 \\
\hline Week 2: May 6-12 & 241 & 150 & 140 & 40 & 18 & 6 & 595 \\
\hline Week 3: May 13-19 & 330 & 150 & 169 & 20 & 14 & 7 & 690 \\
\hline \multicolumn{8}{|c|}{ 40ft Refrigerated } \\
\hline & \multicolumn{6}{|c|}{ Carrier Availability Category Responses } & Total \\
\hline Current Week: Apr 29-May 5 & 0 & . & . & . & . &. & 0 \\
\hline Week 2: May 6-12 & 0 & . & . & . & . & . & 0 \\
\hline Week 3: May 13-19 & 0 & . & . & . & . &. & 0 \\
\hline \multicolumn{8}{|c|}{ 20ft Refrigerated } \\
\hline & \multicolumn{6}{|c|}{ Carrier Availability Category Responses } & Total \\
\hline Current Week: Apr 29-May 5 & . & . & . & . & . & . & 0 \\
\hline Week 2: May 6-12 & . & . & . & . & . & . & 0 \\
\hline Week 3: May 13-19 & . & . & . &. & . & . & 0 \\
\hline
\end{tabular}

Note: "." denotes no response from carriers.

\begin{tabular}{|l|c|c|}
\hline \multicolumn{3}{|c|}{ Six-Month Snapshot of Average Weekly } \\
Throughput \\
June 2012-November 2012 \\
\hline \multirow{2}{*}{ Container Types } & $\begin{array}{c}\text { Average Total Weekly } \\
\text { Throughput* }\end{array}$ & $\begin{array}{c}\text { Range of Individual } \\
\text { Carrier Responses }\end{array}$ \\
\hline $\mathbf{2 0 f t}$ & 58 & $3 . . .17$ \\
$\mathbf{4 0 f t}$ & 43 & $1 \ldots 10$ \\
$\mathbf{4 0 f t}$ High Cubes & 104 & $1 \ldots 50$ \\
$\mathbf{4 0 f t}$ Refrigerated & 0 & $0 \ldots 0$ \\
20ft Refrigerated & 0 & $0 . . .0$ \\
\hline
\end{tabular}

*Data reflect the sum of the individual carriers' average weekly throughput over a 6-month timeframe 


\section{Houston, TX}

\begin{tabular}{|c|c|c|c|c|c|c|c|}
\hline \multicolumn{8}{|c|}{ Houston } \\
\hline \multicolumn{8}{|c|}{ 20ft Dry } \\
\hline & \multicolumn{6}{|c|}{ Carrier Availability Category Responses } & Total \\
\hline Current Week: Apr 29-May 5 & 964 & 541 & 337 & 141 & 134 & 82 & 2,199 \\
\hline Week 2: May 6-12 & 1,059 & 545 & 337 & 156 & 100 & 72 & 2,269 \\
\hline Week 3: May 13-19 & 1,182 & 568 & 337 & 150 & 100 & 65 & 2,402 \\
\hline \multicolumn{8}{|c|}{ 40ft Dry } \\
\hline & \multicolumn{6}{|c|}{ Carrier Availability Category Responses } & Total \\
\hline Current Week: Apr 29-May 5 & 224 & 179 & 127 & 93 & 69 & -245 & 447 \\
\hline Week 2: May 6-12 & 224 & 190 & 143 & 100 & 60 & -199 & 518 \\
\hline Week 3: May 13-19 & 224 & 165 & 215 & 100 & 55 & -210 & 549 \\
\hline \multicolumn{8}{|c|}{ 40ft High Cube } \\
\hline & \multicolumn{6}{|c|}{ Carrier Availability Category Responses } & Total \\
\hline Current Week: Apr 29-May 5 & 2,454 & 480 & 457 & 317 & 146 & 62 & 3,916 \\
\hline Week 2: May 6-12 & 2,454 & 400 & 594 & 397 & 181 & 84 & 4,110 \\
\hline Week 3: May 13-19 & 2,454 & 400 & 626 & 486 & 194 & 70 & 4,230 \\
\hline \multicolumn{8}{|c|}{ 40ft Refrigerated } \\
\hline & \multicolumn{6}{|c|}{ Carrier Availability Category Responses } & Total \\
\hline Current Week: Apr 29-May 5 & 104 & 97 & 37 & 2 & 0 & . & 240 \\
\hline Week 2: May 6-12 & 129 & 113 & 50 & 2 & 0 & . & 294 \\
\hline Week 3: May 13-19 & 154 & 124 & 100 & 2 & 0 & . & 380 \\
\hline \multicolumn{8}{|c|}{ 20ft Refrigerated } \\
\hline & \multicolumn{6}{|c|}{ Carrier Availability Category Responses } & Total \\
\hline Current Week: Apr 29-May 5 & 21 & 3 & 0 & . & . & 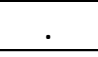 & 24 \\
\hline Week 2: May 6-12 & 22 & 3 & 0 & . & . & . & 25 \\
\hline Week 3: May 13-19 & 24 & 3 & 0 & . & . & . & 27 \\
\hline
\end{tabular}

Note: "." denotes no response from carriers.

\begin{tabular}{|l|c|c|}
\hline \multicolumn{3}{|c|}{ Six-Month Snapshot of Average Weekly } \\
Throughput \\
June 2012-November 2012 \\
\hline \multirow{2}{*}{ Container Types } & $\begin{array}{c}\text { Average Total Weekly } \\
\text { Throughput* }\end{array}$ & $\begin{array}{c}\text { Range of Individual } \\
\text { Carrier Responses }\end{array}$ \\
& 738 & $50 . . .316$ \\
$\mathbf{2 0 f t}$ & 619 & $50 \ldots 126$ \\
$\mathbf{4 0 f t}$ & 725 & $26 \ldots 355$ \\
40ft High Cubes & 31 & $0 . .12$ \\
40ft Refrigerated & 1 & $0 . .1$ \\
\hline 20ft Refrigerated &
\end{tabular}

*Data reflect the sum of the individual carriers' average weekly throughput over a 6-month timeframe 


\section{Kansas City, MO}

\begin{tabular}{|c|c|c|c|c|c|c|c|}
\hline \multicolumn{8}{|c|}{ Kansas City } \\
\hline \multicolumn{8}{|c|}{ 20ft Dry } \\
\hline & \multicolumn{6}{|c|}{ Carrier Availability Category Responses } & Total \\
\hline Current Week: Apr 29-May 5 & 124 & 63 & 50 & 37 & 29 & 5 & 308 \\
\hline Week 2: May 6-12 & 124 & 22 & 62 & 30 & 48 & 3 & 289 \\
\hline Week 3: May 13-19 & 124 & 38 & 74 & 30 & 60 & 3 & 329 \\
\hline \multicolumn{8}{|c|}{ 40ft Dry } \\
\hline & \multicolumn{6}{|c|}{ Carrier Availability Category Responses } & Total \\
\hline Current Week: Apr 29-May 5 & 114 & 57 & 27 & 9 & 5 & -36 & 176 \\
\hline Week 2: May 6-12 & 142 & 77 & 26 & 4 & 0 & -36 & 213 \\
\hline Week 3: May 13-19 & 170 & 89 & 44 & 6 & 0 & -36 & 273 \\
\hline \multicolumn{8}{|c|}{ 40ft High Cube } \\
\hline & \multicolumn{6}{|c|}{ Carrier Availability Category Responses } & Total \\
\hline Current Week: Apr 29-May 5 & 148 & 96 & 80 & 51 & 2 & -170 & 207 \\
\hline Week 2: May 6-12 & 181 & 80 & 97 & -6 & 0 & -170 & 182 \\
\hline Week 3: May 13-19 & 211 & 40 & 107 & 11 & 2 & -170 & 201 \\
\hline \multicolumn{8}{|c|}{ 40ft Refrigerated } \\
\hline & \multicolumn{6}{|c|}{ Carrier Availability Category Responses } & Total \\
\hline Current Week: Apr 29-May 5 & 43 & 26 & 0 &. &. & . & 69 \\
\hline Week 2: May 6-12 & 34 & 28 & 0 &. & . & . & 62 \\
\hline Week 3: May 13-19 & 25 & 30 & 0 & . & . & . & 55 \\
\hline \multicolumn{8}{|c|}{ 20ft Refrigerated } \\
\hline & \multicolumn{6}{|c|}{ Carrier Availability Category Responses } & Total \\
\hline Current Week: Apr 29-May 5 & 1 & 1 & . & . & . & . & 2 \\
\hline Week 2: May 6-12 & 1 & 1 & . & . & . & . & 2 \\
\hline Week 3: May 13-19 & 2 & 1 & . & . &. & . & 3 \\
\hline
\end{tabular}

Note: "." denotes no response from carriers.

\begin{tabular}{|l|l|l|}
\hline \multicolumn{3}{|c|}{ Six-Month Snapshot of Average Weekly } \\
Throughput \\
June 2012-November 2012 \\
\hline Container Types & $\begin{array}{c}\text { Average Total Weekly } \\
\text { Throughput* }\end{array}$ & $\begin{array}{c}\text { Range of Individual } \\
\text { Carrier Responses }\end{array}$ \\
\hline 20ft & 171 & $7 \ldots 60$ \\
40ft & 196 & $9 . .75$ \\
40ft High Cubes & 198 & $14 . .59$ \\
40ft Refrigerated & 1 & $0 \ldots 1$ \\
20ft Refrigerated & 0 & $0 . .0$ \\
\hline
\end{tabular}

*Data reflect the sum of the individual carriers' average weekly throughput over a 6-month timeframe 


\begin{tabular}{|c|c|c|c|c|c|c|c|}
\hline \multicolumn{8}{|c|}{ Los Angeles and Long Beach } \\
\hline \multicolumn{8}{|c|}{ 20ft Dry } \\
\hline & \multicolumn{6}{|c|}{ Carrier Availability Category Responses } & Total \\
\hline Current Week: Apr 29-May 5 & 1,248 & 1,168 & 896 & 841 & 485 & -13 & 4,625 \\
\hline Week 2: May 6-12 & 1,248 & 1,533 & 900 & 1,000 & 534 & 101 & 5,316 \\
\hline Week 3: May 13-19 & 1,248 & 1,739 & 1,027 & 1,000 & 658 & 45 & 5,717 \\
\hline \multicolumn{8}{|c|}{ 40ft Dry } \\
\hline & \multicolumn{6}{|c|}{ Carrier Availability Category Responses } & Total \\
\hline Current Week: Apr 29-May 5 & 978 & 958 & 896 & 838 & 732 & 430 & 4,832 \\
\hline Week 2: May 6-12 & 1,186 & 958 & 1,110 & 898 & 600 & 407 & 5,159 \\
\hline Week 3: May 13-19 & 1,363 & 958 & 1,318 & 845 & 600 & 311 & 5,395 \\
\hline \multicolumn{8}{|c|}{ 40ft High Cube } \\
\hline & \multicolumn{6}{|c|}{ Carrier Availability Category Responses } & Total \\
\hline Current Week: Apr 29-May 5 & 7,588 & 2,809 & 2,257 & 1,674 & 797 & 576 & 15,701 \\
\hline Week 2: May 6-12 & 7,588 & 1,500 & 2,226 & 1,800 & 985 & 570 & 14,669 \\
\hline Week 3: May 13-19 & 7,588 & 1,500 & 2,661 & 1,780 & 1,095 & 501 & 15,125 \\
\hline \multicolumn{8}{|c|}{ 40ft Refrigerated } \\
\hline & \multicolumn{6}{|c|}{ Carrier Availability Category Responses } & Total \\
\hline Current Week: Apr 29-May 5 & 1,237 & 851 & 161 & 155 & 75 & 57 & 2,536 \\
\hline Week 2: May 6-12 & 600 & 866 & 53 & 212 & 75 & -15 & 1,791 \\
\hline Week 3: May 13-19 & 600 & 896 & 101 & 175 & 75 & -32 & 1,815 \\
\hline \multicolumn{8}{|c|}{ 20ft Refrigerated } \\
\hline & \multicolumn{6}{|c|}{ Carrier Availability Category Responses } & Total \\
\hline Current Week: Apr 29-May 5 & 124 & 34 & 10 & . & . & . & 168 \\
\hline Week 2: May 6-12 & 137 & 37 & 10 & . & . & . & 184 \\
\hline Week 3: May 13-19 & 189 & 40 & 10 & . & . & . & 239 \\
\hline
\end{tabular}

Note: "." denotes no response from carriers.

\begin{tabular}{|l|c|c|}
\hline \multicolumn{3}{|c|}{ Six-Month Snapshot of Average Weekly } \\
Throughput \\
June 2012-November 2012 \\
\hline \multirow{2}{*}{ Container Types } & $\begin{array}{c}\text { Average Total Weekly } \\
\text { Throughput* }\end{array}$ & $\begin{array}{c}\text { Range of Individual } \\
\text { Carrier Responses }\end{array}$ \\
\hline $\mathbf{2 0 f t}$ & 3,827 & $46 . .2500$ \\
$\mathbf{4 0 f t}$ & 4,744 & $83 . . .3000$ \\
40ft High Cubes & 7,085 & $203 \ldots 3300$ \\
40ft Refrigerated & 684 & $21 . . .300$ \\
20ft Refrigerated & 64 & $1 . . .50$ \\
\hline
\end{tabular}

*Data reflect the sum of the individual carriers' average weekly throughput over a 6-month timeframe 


\section{Memphis, TN}

\begin{tabular}{|c|c|c|c|c|c|c|c|}
\hline \multicolumn{8}{|c|}{ Memphis } \\
\hline \multicolumn{8}{|c|}{ 20ft Dry } \\
\hline & \multicolumn{6}{|c|}{ Carrier Availability Category Responses } & Total \\
\hline Current Week: Apr 29-May 5 & 257 & 215 & 156 & 115 & 12 & -79 & 676 \\
\hline Week 2: May 6-12 & 257 & 267 & 206 & 100 & 15 & -58 & 787 \\
\hline Week 3: May 13-19 & 257 & 310 & 222 & 100 & 10 & -39 & 860 \\
\hline \multicolumn{8}{|c|}{ 40ft Dry } \\
\hline & \multicolumn{6}{|c|}{ Carrier Availability Category Responses } & Total \\
\hline Current Week: Apr 29-May 5 & 488 & 183 & 134 & 115 & 17 & 14 & 951 \\
\hline Week 2: May 6-12 & 668 & 177 & 134 & 100 & 20 & 31 & 1,130 \\
\hline Week 3: May 13-19 & 846 & 188 & 134 & 100 & 18 & 57 & 1,343 \\
\hline \multicolumn{8}{|c|}{ 40ft High Cube } \\
\hline & \multicolumn{6}{|c|}{ Carrier Availability Category Responses } & Total \\
\hline Current Week: Apr 29-May 5 & 1,312 & 510 & 159 & 96 & 31 & -47 & 2,061 \\
\hline Week 2: May 6-12 & 1,312 & 754 & 219 & 100 & 45 & 0 & 2,430 \\
\hline Week 3: May 13-19 & 1,312 & 923 & 271 & 100 & 40 & 51 & 2,697 \\
\hline \multicolumn{8}{|c|}{ 40ft Refrigerated } \\
\hline & \multicolumn{6}{|c|}{ Carrier Availability Category Responses } & Total \\
\hline Current Week: Apr 29-May 5 & 8 & 4 & 0 & . & . & . & 12 \\
\hline Week 2: May 6-12 & 13 & 6 & 0 & . & . & . & 19 \\
\hline Week 3: May 13-19 & 17 & 8 & 0 & . & . & . & 25 \\
\hline \multicolumn{8}{|c|}{ 20ft Refrigerated } \\
\hline & \multicolumn{6}{|c|}{ Carrier Availability Category Responses } & Total \\
\hline Current Week: Apr 29-May 5 & . & . & . & . & . & . & 0 \\
\hline Week 2: May 6-12 & . & . & . & . & . & . & 0 \\
\hline Week 3: May 13-19 & . & . & . & . & . & . & 0 \\
\hline
\end{tabular}

Note: "." denotes no response from carriers.

\begin{tabular}{|l|c|c|}
\hline \multicolumn{3}{|c|}{ Six-Month Snapshot of Average Weekly } \\
Throughput \\
June 2012-November 2012 \\
\hline Container Types & $\begin{array}{c}\text { Average Total Weekly } \\
\text { Throughput* }\end{array}$ & $\begin{array}{c}\text { Range of Individual } \\
\text { Carrier Responses }\end{array}$ \\
\hline $\mathbf{2 0 f t}$ & 558 & $11 \ldots 300$ \\
$\mathbf{4 0 f t}$ & 430 & $11 \ldots 200$ \\
40ft High Cubes & 884 & $9 . .600$ \\
40ft Refrigerated & 2 & $0 . . .2$ \\
20ft Refrigerated & 0 & $0 . . .0$ \\
\hline
\end{tabular}

*Data reflect the sum of the individual carriers' average weekly throughput over a 6-month timeframe 


\section{Minneapolis, MN}

\begin{tabular}{|c|c|c|c|c|c|c|c|}
\hline \multicolumn{8}{|c|}{ Minneapolis } \\
\hline \multicolumn{8}{|c|}{ 20ft Dry } \\
\hline & \multicolumn{6}{|c|}{ Carrier Availability Category Responses } & Total \\
\hline Current Week: Apr 29-May 5 & 63 & 47 & 27 & 1 & -6 & -75 & 57 \\
\hline Week 2: May 6-12 & 6 & 47 & 51 & 0 & 6 & -92 & 18 \\
\hline Week 3: May 13-19 & -76 & 47 & 60 & 25 & -4 & -106 & -54 \\
\hline \multicolumn{8}{|c|}{ 40ft Dry } \\
\hline & \multicolumn{6}{|c|}{ Carrier Availability Category Responses } & Total \\
\hline Current Week: Apr 29-May 5 & 152 & 104 & 87 & 60 & 14 & 3 & 420 \\
\hline Week 2: May 6-12 & 152 & 120 & 82 & 38 & 30 & 5 & 427 \\
\hline Week 3: May 13-19 & 152 & 118 & 75 & 6 & 30 & 8 & 389 \\
\hline \multicolumn{8}{|c|}{ 40ft High Cube } \\
\hline & \multicolumn{6}{|c|}{ Carrier Availability Category Responses } & Total \\
\hline Current Week: Apr 29-May 5 & 300 & 86 & 84 & 74 & 69 & -8 & 605 \\
\hline Week 2: May 6-12 & 300 & 75 & 50 & 55 & 79 & 12 & 571 \\
\hline Week 3: May 13-19 & 300 & 63 & 40 & 47 & 113 & 5 & 568 \\
\hline \multicolumn{8}{|c|}{ 40ft Refrigerated } \\
\hline & \multicolumn{6}{|c|}{ Carrier Availability Category Responses } & Total \\
\hline Current Week: Apr 29-May 5 & 0 & -14 & . & . & . & . & -14 \\
\hline Week 2: May 6-12 & 0 & -20 & . &. & . & . & -20 \\
\hline Week 3: May 13-19 & 0 & -24 & . & . & . & . & -24 \\
\hline \multicolumn{8}{|c|}{ 20ft Refrigerated } \\
\hline & \multicolumn{6}{|c|}{ Carrier Availability Category Responses } & Total \\
\hline Current Week: Apr 29-May 5 & . & . & . & . & . & . & 0 \\
\hline Week 2: May 6-12 & . & . & . & . & . & . & 0 \\
\hline Week 3: May 13-19 & . & . & . &. & . & . & 0 \\
\hline
\end{tabular}

Note: "." denotes no response from carriers.

\section{Six-Month Snapshot of Average Weekly} Throughput

June 2012-November 2012

\begin{tabular}{|l|c|c|}
\hline Container Types & $\begin{array}{c}\text { Average Total Weekly } \\
\text { Throughput* }\end{array}$ & $\begin{array}{c}\text { Range of Individual } \\
\text { Carrier Responses }\end{array}$ \\
\hline $\mathbf{2 0 f t}$ & 200 & $3 \ldots 102$ \\
$\mathbf{4 0 f t}$ & 181 & $1 \ldots 80$ \\
$\mathbf{4 0 f t}$ High Cubes & 223 & $2 \ldots 144$ \\
40ft Refrigerated & 4 & $0 . . .4$ \\
20ft Refrigerated & 1 & $0 . . .1$ \\
\hline
\end{tabular}

*Data reflect the sum of the individual carriers' average weekly throughput over a 6-month timeframe 


\section{New Orleans, LA}

\begin{tabular}{|c|c|c|c|c|c|c|c|}
\hline \multicolumn{8}{|c|}{ New Orleans } \\
\hline \multicolumn{8}{|c|}{ 20ft Dry } \\
\hline & \multicolumn{6}{|c|}{ Carrier Availability Category Responses } & Total \\
\hline Current Week: Apr 29-May 5 & 404 & 90 & 68 & 18 & 7 & 1 & 588 \\
\hline Week 2: May 6-12 & 397 & 60 & 80 & 18 & 0 & 1 & 556 \\
\hline Week 3: May 13-19 & 286 & 45 & 88 & 18 & 10 & 1 & 448 \\
\hline \multicolumn{8}{|c|}{ 40ft Dry } \\
\hline & \multicolumn{6}{|c|}{ Carrier Availability Category Responses } & Total \\
\hline Current Week: Apr 29-May 5 & 92 & 58 & 21 & 8 & 6 & 1 & 186 \\
\hline Week 2: May 6-12 & 73 & 40 & 21 & 8 & 0 & 2 & 144 \\
\hline Week 3: May 13-19 & 53 & 25 & 21 & 8 & 10 & 2 & 119 \\
\hline \multicolumn{8}{|c|}{ 40ft High Cube } \\
\hline & \multicolumn{6}{|c|}{ Carrier Availability Category Responses } & Total \\
\hline Current Week: Apr 29-May 5 & 232 & 91 & 40 & 26 & 21 & 2 & 412 \\
\hline Week 2: May 6-12 & 119 & 90 & 38 & 26 & 10 & 2 & 285 \\
\hline Week 3: May 13-19 & 1 & 103 & 41 & 26 & 10 & 2 & 183 \\
\hline \multicolumn{8}{|c|}{ 40ft Refrigerated } \\
\hline & \multicolumn{6}{|c|}{ Carrier Availability Category Responses } & Total \\
\hline Current Week: Apr 29-May 5 & 58 & 0 & -14 & . & $\cdot$ & . & 44 \\
\hline Week 2: May 6-12 & 57 & 0 & -26 & . & . & . & 31 \\
\hline Week 3: May 13-19 & 47 & 0 & -38 & . & . & . & 9 \\
\hline \multicolumn{8}{|c|}{ 20ft Refrigerated } \\
\hline & \multicolumn{6}{|c|}{ Carrier Availability Category Responses } & Total \\
\hline Current Week: Apr 29-May 5 & 5 & 4 & . & . & . & . & 9 \\
\hline Week 2: May 6-12 & 5 & 3 & . & . & . & . & 8 \\
\hline Week 3: May 13-19 & 5 & -2 & . &. & . & . & 3 \\
\hline
\end{tabular}

Note: "." denotes no response from carriers.

\begin{tabular}{|l|c|c|}
\hline \multicolumn{3}{|c|}{ Six-Month Snapshot of Average Weekly } \\
Throughput \\
June 2012-November 2012 \\
\hline Container Types & $\begin{array}{c}\text { Average Total Weekly } \\
\text { Throughput* }\end{array}$ & $\begin{array}{c}\text { Range of Individual } \\
\text { Carrier Responses }\end{array}$ \\
\hline $\mathbf{2 0 f t}$ & 246 & $5 \ldots 158$ \\
$\mathbf{4 0 f t}$ & 157 & $5 \ldots .80$ \\
40ft High Cubes & 221 & $5 \ldots 139$ \\
40ft Refrigerated & 1 & $0 \ldots 1$ \\
$\mathbf{2 0 f t}$ Refrigerated & 5 & $0 \ldots 5$ \\
\hline
\end{tabular}

*Data reflect the sum of the individual carriers' average weekly throughput over a 6-month timeframe 


\section{New York, NY}

\begin{tabular}{|c|c|c|c|c|c|c|c|}
\hline \multicolumn{8}{|c|}{ New York } \\
\hline \multicolumn{8}{|c|}{ 20ft Dry } \\
\hline & \multicolumn{6}{|c|}{ Carrier Availability Category Responses } & Total \\
\hline Current Week: Apr 29-May 5 & 1,279 & 1,197 & 910 & 690 & 662 & 328 & 5,066 \\
\hline Week 2: May 6-12 & 1,614 & 1,155 & 800 & 690 & 832 & 278 & 5,369 \\
\hline Week 3: May 13-19 & 1,954 & 1,022 & 800 & 690 & 1,095 & 300 & 5,861 \\
\hline \multicolumn{8}{|c|}{ 40ft Dry } \\
\hline & \multicolumn{6}{|c|}{ Carrier Availability Category Responses } & Total \\
\hline Current Week: Apr 29-May 5 & 664 & 645 & 628 & 512 & 200 & -9 & 2,640 \\
\hline Week 2: May 6-12 & 758 & 884 & 706 & 800 & 200 & 28 & 3,376 \\
\hline Week 3: May 13-19 & 814 & 1,132 & 875 & 800 & 200 & 28 & 3,849 \\
\hline \multicolumn{8}{|c|}{ 40ft High Cube } \\
\hline & \multicolumn{6}{|c|}{ Carrier Availability Category Responses } & Total \\
\hline Current Week: Apr 29-May 5 & 2,716 & 2,308 & 1,516 & 1,378 & 949 & 242 & 9,109 \\
\hline Week 2: May 6-12 & 2,716 & 2,451 & 1,984 & 1,639 & 1,000 & 253 & 10,043 \\
\hline Week 3: May 13-19 & 2,716 & 2,944 & 2,494 & 1,981 & 1,000 & 235 & 11,370 \\
\hline \multicolumn{8}{|c|}{ 40ft Refrigerated } \\
\hline & \multicolumn{6}{|c|}{ Carrier Availability Category Responses } & Total \\
\hline Current Week: Apr 29-May 5 & 561 & 242 & 206 & 80 & 10 & 9 & 1,108 \\
\hline Week 2: May 6-12 & 638 & 355 & 299 & 100 & 10 & 62 & 1,464 \\
\hline Week 3: May 13-19 & 724 & 443 & 403 & 100 & 10 & 60 & 1,740 \\
\hline \multicolumn{8}{|c|}{ 20ft Refrigerated } \\
\hline & \multicolumn{6}{|c|}{ Carrier Availability Category Responses } & Total \\
\hline Current Week: Apr 29-May 5 & 21 & 10 & 5 & $\dot{\varphi}$ & . & . & 36 \\
\hline Week 2: May 6-12 & 22 & 10 & 5 & . & . & . & 37 \\
\hline Week 3: May 13-19 & 24 & 7 & 5 & $\cdot$ & . & . & 36 \\
\hline
\end{tabular}

Note: "." denotes no response from carriers.

Six-Month Snapshot of Average Weekly Throughput

June 2012-November 2012

\begin{tabular}{|l|c|c|}
\hline Container Types & $\begin{array}{c}\text { Average Total Weekly } \\
\text { Throughput* }\end{array}$ & $\begin{array}{c}\text { Range of Individual } \\
\text { Carrier Responses }\end{array}$ \\
\hline 20ft & 714 & $3 \ldots 150$ \\
40ft & 901 & $56 \ldots 250$ \\
40ft High Cubes & 1,539 & $87 . . .400$ \\
40ft Refrigerated & 113 & $0 \ldots 36$ \\
20ft Refrigerated & 17 & $0 \ldots 10$ \\
\hline
\end{tabular}

*Data reflect the sum of the individual carriers' average weekly throughput over a 6-month timeframe 


\section{Norfolk, VA}

\begin{tabular}{|c|c|c|c|c|c|c|c|}
\hline \multicolumn{8}{|c|}{ Norfolk } \\
\hline \multicolumn{8}{|c|}{ 20ft Dry } \\
\hline & \multicolumn{6}{|c|}{ Carrier Availability Category Responses } & Total \\
\hline Current Week: Apr 29-May 5 & 491 & 442 & 331 & 317 & 183 & 21 & 1,785 \\
\hline Week 2: May 6-12 & 400 & 442 & 364 & 320 & 172 & 23 & 1,721 \\
\hline Week 3: May 13-19 & 300 & 442 & 385 & 413 & 174 & 20 & 1,734 \\
\hline \multicolumn{8}{|c|}{ 40ft Dry } \\
\hline & \multicolumn{6}{|c|}{ Carrier Availability Category Responses } & Total \\
\hline Current Week: Apr 29-May 5 & 321 & 305 & 294 & 107 & 32 & -48 & 1,011 \\
\hline Week 2: May 6-12 & 300 & 471 & 294 & 135 & 58 & -13 & 1,245 \\
\hline Week 3: May 13-19 & 200 & 534 & 294 & 136 & 74 & -28 & 1,210 \\
\hline \multicolumn{8}{|c|}{ 40ft High Cube } \\
\hline & \multicolumn{6}{|c|}{ Carrier Availability Category Responses } & Total \\
\hline Current Week: Apr 29-May 5 & 2,918 & 1,211 & 763 & 596 & 179 & -58 & 5,609 \\
\hline Week 2: May 6-12 & 2,918 & 800 & 866 & 718 & 206 & -50 & 5,458 \\
\hline Week 3: May 13-19 & 2,918 & 500 & 849 & 818 & 232 & -52 & 5,265 \\
\hline \multicolumn{8}{|c|}{ 40ft Refrigerated } \\
\hline & \multicolumn{6}{|c|}{ Carrier Availability Category Responses } & Total \\
\hline Current Week: Apr 29-May 5 & 141 & 122 & 58 & 18 & 10 & -61 & 288 \\
\hline Week 2: May 6-12 & 50 & 106 & 39 & 25 & 10 & -16 & 214 \\
\hline Week 3: May 13-19 & 50 & 104 & 44 & -88 & 10 & -42 & 78 \\
\hline \multicolumn{8}{|c|}{ 20ft Refrigerated } \\
\hline & \multicolumn{6}{|c|}{ Carrier Availability Category Responses } & Total \\
\hline Current Week: Apr 29-May 5 & 12 & 6 & 1 & . & . & . & 19 \\
\hline Week 2: May 6-12 & 12 & 6 & 1 & . & . & $\cdot$ & 19 \\
\hline Week 3: May 13-19 & 13 & 6 & 1 & . & . & & 20 \\
\hline
\end{tabular}

Note: "." denotes no response from carriers.

Six-Month Snapshot of Average Weekly Throughput

June 2012-November 2012

\begin{tabular}{|l|c|c|}
\hline Container Types & $\begin{array}{c}\text { Average Total Weekly } \\
\text { Throughput* }\end{array}$ & $\begin{array}{c}\text { Range of Individual } \\
\text { Carrier Responses }\end{array}$ \\
\hline 20ft & 338 & $1 \ldots 175$ \\
$\mathbf{4 0 f t}$ & 797 & $8 \ldots 200$ \\
40ft High Cubes & 1,035 & $74 \ldots 300$ \\
40ft Refrigerated & 139 & $0 \ldots 58$ \\
20ft Refrigerated & 6 & $0 . . .5$ \\
\hline
\end{tabular}

*Data reflect the sum of the individual carriers' average weekly throughput over a 6-month timeframe 


\section{Oakland, CA}

\begin{tabular}{|c|c|c|c|c|c|c|c|}
\hline \multicolumn{8}{|c|}{ Oakland } \\
\hline \multicolumn{8}{|c|}{ 20ft Dry } \\
\hline & \multicolumn{6}{|c|}{ Carrier Availability Category Responses } & Total \\
\hline Current Week: Apr 29-May 5 & 381 & 379 & 236 & 184 & 65 & -2 & 1,243 \\
\hline Week 2: May 6-12 & 231 & 354 & 114 & 100 & 65 & 20 & 884 \\
\hline Week 3: May 13-19 & 210 & 316 & 117 & 100 & 65 & 33 & 841 \\
\hline \multicolumn{8}{|c|}{ 40ft Dry } \\
\hline & \multicolumn{6}{|c|}{ Carrier Availability Category Responses } & Total \\
\hline Current Week: Apr 29-May 5 & 445 & 166 & 73 & 54 & 29 & -65 & 702 \\
\hline Week 2: May 6-12 & 422 & 131 & -67 & 54 & 50 & -74 & 516 \\
\hline Week 3: May 13-19 & 408 & 120 & -34 & 54 & 100 & -55 & 593 \\
\hline \multicolumn{8}{|c|}{ 40ft High Cube } \\
\hline & \multicolumn{6}{|c|}{ Carrier Availability Category Responses } & Total \\
\hline Current Week: Apr 29-May 5 & 940 & 553 & 174 & 15 & -74 & -198 & 1,410 \\
\hline Week 2: May 6-12 & 979 & 380 & -494 & 29 & 0 & -198 & 696 \\
\hline Week 3: May 13-19 & 1,014 & 343 & -750 & 70 & 0 & -198 & 479 \\
\hline \multicolumn{8}{|c|}{ 40ft Refrigerated } \\
\hline & \multicolumn{6}{|c|}{ Carrier Availability Category Responses } & Total \\
\hline Current Week: Apr 29-May 5 & 348 & 339 & 47 & 30 & 11 & -105 & 670 \\
\hline Week 2: May 6-12 & 200 & 208 & 51 & 30 & -9 & -48 & 432 \\
\hline Week 3: May 13-19 & 200 & 214 & 83 & 30 & -27 & -84 & 416 \\
\hline \multicolumn{8}{|c|}{ 20ft Refrigerated } \\
\hline & \multicolumn{6}{|c|}{ Carrier Availability Category Responses } & Total \\
\hline Current Week: Apr 29-May 5 & 22 & 10 & 7 & . & . & . & 39 \\
\hline Week 2: May 6-12 & 23 & 10 & 4 & . & . & . & 37 \\
\hline Week 3: May 13-19 & 23 & 10 & 3 & . & . & . & 36 \\
\hline
\end{tabular}

Note: "." denotes no response from carriers.

\begin{tabular}{|l|c|c|}
\hline \multicolumn{3}{|c|}{ Six-Month Snapshot of Average Weekly } \\
Throughput \\
June 2012-November 2012 \\
\hline \multirow{2}{*}{ Container Types } & $\begin{array}{c}\text { Average Total Weekly } \\
\text { Throughput* }\end{array}$ & $\begin{array}{c}\text { Range of Individual } \\
\text { Carrier Responses }\end{array}$ \\
\hline $\mathbf{2 0 f t}$ & 853 & $26 \ldots 250$ \\
$\mathbf{4 0 f t}$ & 899 & $14 . . .300$ \\
40ft High Cubes & 1,199 & $34 \ldots 400$ \\
40ft Refrigerated & 358 & $26 \ldots 120$ \\
20ft Refrigerated & 24 & $0 . .8$ \\
\hline
\end{tabular}

*Data reflect the sum of the individual carriers' average weekly throughput over a 6-month timeframe 


\section{Savannah, GA}

\begin{tabular}{|c|c|c|c|c|c|c|c|}
\hline \multicolumn{8}{|c|}{ Savannah } \\
\hline \multicolumn{8}{|c|}{ 20ft Dry } \\
\hline & \multicolumn{6}{|c|}{ Carrier Availability Category Responses } & Total \\
\hline Current Week: Apr 29-May 5 & 674 & 365 & 275 & 263 & 86 & 72 & 1,735 \\
\hline Week 2: May 6-12 & 600 & 466 & 371 & 265 & 86 & 59 & 1,847 \\
\hline Week 3: May 13-19 & 60 & 568 & 516 & 233 & 86 & 42 & 1,505 \\
\hline \multicolumn{8}{|c|}{ 40ft Dry } \\
\hline & \multicolumn{6}{|c|}{ Carrier Availability Category Responses } & Total \\
\hline Current Week: Apr 29-May 5 & 652 & 542 & 129 & 31 & 9 & -5 & 1,358 \\
\hline Week 2: May 6-12 & 658 & 542 & 100 & -60 & 16 & -24 & 1,232 \\
\hline Week 3: May 13-19 & 703 & 542 & 100 & 123 & 13 & -20 & 1,461 \\
\hline \multicolumn{8}{|c|}{ 40ft High Cube } \\
\hline & \multicolumn{6}{|c|}{ Carrier Availability Category Responses } & Total \\
\hline Current Week: Apr 29-May 5 & 1,448 & 777 & 768 & 232 & 95 & -106 & 3,214 \\
\hline Week 2: May 6-12 & 1,448 & 1,100 & 839 & 107 & 100 & -66 & 3,528 \\
\hline Week 3: May 13-19 & 1,448 & 1,406 & 892 & 134 & 300 & -70 & 4,110 \\
\hline \multicolumn{8}{|c|}{ 40ft Refrigerated } \\
\hline & \multicolumn{6}{|c|}{ Carrier Availability Category Responses } & Total \\
\hline Current Week: Apr 29-May 5 & 163 & 43 & 39 & 16 & 10 & -13 & 258 \\
\hline Week 2: May 6-12 & 100 & -15 & 47 & -29 & 10 & -10 & 103 \\
\hline Week 3: May 13-19 & 50 & -37 & 56 & -27 & 10 & -26 & 26 \\
\hline \multicolumn{8}{|c|}{ 20ft Refrigerated } \\
\hline & \multicolumn{6}{|c|}{ Carrier Availability Category Responses } & Total \\
\hline Current Week: Apr 29-May 5 & 18 & 11 & 1 & . & . & . & 30 \\
\hline Week 2: May 6-12 & 18 & 11 & 1 & . & . & . & 30 \\
\hline Week 3: May 13-19 & 19 & 11 & 1 & . & . & 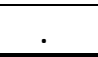 & 31 \\
\hline
\end{tabular}

Note: "." denotes no response from carriers.

\begin{tabular}{|l|c|c|}
\hline \multicolumn{3}{|c|}{ Six-Month Snapshot of Average Weekly } \\
Throughput \\
June 2012-November 2012 \\
\hline \multirow{2}{*}{ Container Types } & $\begin{array}{c}\text { Average Total Weekly } \\
\text { Throughput* }\end{array}$ & $\begin{array}{c}\text { Range of Individual } \\
\text { Carrier Responses }\end{array}$ \\
\hline $\mathbf{2 0 f t}$ & 449 & $0 \ldots 150$ \\
$\mathbf{4 0 f t}$ & 982 & $0 . .409$ \\
$\mathbf{4 0 f t}$ High Cubes & 1,450 & $21 . .385$ \\
$\mathbf{4 0 f t}$ Refrigerated & 221 & $0 . .80$ \\
20ft Refrigerated & 4 & $0 \ldots 2$ \\
\hline
\end{tabular}

*Data reflect the sum of the individual carriers' average weekly throughput over a 6-month timeframe 


\section{Seattle, WA}

\begin{tabular}{|c|c|c|c|c|c|c|c|}
\hline \multicolumn{8}{|c|}{ Seattle } \\
\hline \multicolumn{8}{|c|}{ 20ft Dry } \\
\hline & \multicolumn{6}{|c|}{ Carrier Availability Category Responses } & Total \\
\hline Current Week: Apr 29-May 5 & 468 & 316 & 206 & 165 & -132 & . & 1,023 \\
\hline Week 2: May 6-12 & 513 & 316 & 200 & 206 & -209 & . & 1,026 \\
\hline Week 3: May 13-19 & 553 & 316 & 200 & 243 & -232 & . & 1,080 \\
\hline \multicolumn{8}{|c|}{ 40ft Dry } \\
\hline & \multicolumn{6}{|c|}{ Carrier Availability Category Responses } & Total \\
\hline Current Week: Apr 29-May 5 & 446 & 151 & 116 & 81 & 14 & . & 808 \\
\hline Week 2: May 6-12 & 446 & 160 & 200 & 47 & 1 & . & 854 \\
\hline Week 3: May 13-19 & 446 & 163 & 200 & 22 & -9 & . & 822 \\
\hline \multicolumn{8}{|c|}{ 40ft High Cube } \\
\hline & \multicolumn{6}{|c|}{ Carrier Availability Category Responses } & Total \\
\hline Current Week: Apr 29-May 5 & 606 & -12 & -23 & -68 & -138 & . & 365 \\
\hline Week 2: May 6-12 & 606 & -35 & -107 & 0 & -179 & . & 285 \\
\hline Week 3: May 13-19 & 606 & -112 & -179 & 50 & -222 & . & 143 \\
\hline \multicolumn{8}{|c|}{ 40ft Refrigerated } \\
\hline & \multicolumn{6}{|c|}{ Carrier Availability Category Responses } & Total \\
\hline Current Week: Apr 29-May 5 & 884 & 680 & 114 & 26 & 20 & . & 1,724 \\
\hline Week 2: May 6-12 & 400 & 743 & 4 & 29 & 20 & . & 1,196 \\
\hline Week 3: May 13-19 & 400 & 780 & -106 & 24 & 20 & . & 1,118 \\
\hline \multicolumn{8}{|c|}{ 20ft Refrigerated } \\
\hline & \multicolumn{6}{|c|}{ Carrier Availability Category Responses } & Total \\
\hline Current Week: Apr 29-May 5 & 40 & 21 & 1 & . & . & . & 62 \\
\hline Week 2: May 6-12 & 47 & 23 & 1 & . & . & . & 71 \\
\hline Week 3: May 13-19 & 51 & 25 & 1 & . & . & . & 77 \\
\hline
\end{tabular}

Note: "." denotes no response from carriers.

\begin{tabular}{|l|c|c|}
\hline \multicolumn{3}{|c|}{ Six-Month Snapshot of Average Weekly } \\
Throughput \\
June 2012-November 2012 \\
\hline Container Types & $\begin{array}{c}\text { Average Total Weekly } \\
\text { Throughput* }\end{array}$ & $\begin{array}{c}\text { Range of Individual } \\
\text { Carrier Responses }\end{array}$ \\
\hline 20ft & 172 & $29 . . .70$ \\
40ft & 263 & $7 \ldots 91$ \\
40ft High Cubes & 462 & $104 \ldots 148$ \\
40ft Refrigerated & 172 & $0 \ldots 122$ \\
20ft Refrigerated & 7 & $0 . . .5$ \\
\hline
\end{tabular}

*Data reflect the sum of the individual carriers' average weekly throughput over a 6-month timeframe 


\section{Tacoma, WA}

\begin{tabular}{|c|c|c|c|c|c|c|}
\hline \multicolumn{7}{|c|}{ Tacoma } \\
\hline \multicolumn{7}{|c|}{ 20ft Dry } \\
\hline & \multicolumn{5}{|c|}{ Carrier Availability Category Responses } & Total \\
\hline Current Week: Apr 29-May 5 & 113 & 62 & 40 & -25 & . & 190 \\
\hline Week 2: May 6-12 & 100 & 62 & 40 & -117 & . & 85 \\
\hline Week 3: May 13-19 & 120 & 62 & 80 & -211 & . & 51 \\
\hline \multicolumn{7}{|c|}{ 40ft Dry } \\
\hline & \multicolumn{5}{|c|}{ Carrier Availability Category Responses } & Total \\
\hline Current Week: Apr 29-May 5 & 99 & 69 & 38 & -35 & . & 171 \\
\hline Week 2: May 6-12 & 99 & -57 & 40 & -20 & . & 62 \\
\hline Week 3: May 13-19 & 99 & -6 & 80 & -13 & . & 160 \\
\hline \multicolumn{7}{|c|}{ 40ft High Cube } \\
\hline & \multicolumn{5}{|c|}{ Carrier Availability Category Responses } & Total \\
\hline Current Week: Apr 29-May 5 & 774 & 214 & 130 & -116 & . & 1,002 \\
\hline Week 2: May 6-12 & -100 & 214 & 100 & 51 & . & 265 \\
\hline Week 3: May 13-19 & -436 & 214 & 100 & 65 & . & -57 \\
\hline \multicolumn{7}{|c|}{ 40ft Refrigerated } \\
\hline & \multicolumn{5}{|c|}{ Carrier Availability Category Responses } & Total \\
\hline Current Week: Apr 29-May 5 & 236 & 235 & 5 & -382 & . & 94 \\
\hline Week 2: May 6-12 & 15 & 206 & 5 & -372 & . & -146 \\
\hline Week 3: May 13-19 & 20 & 177 & 5 & -324 & . & -122 \\
\hline \multicolumn{7}{|c|}{ 20ft Refrigerated } \\
\hline & \multicolumn{5}{|c|}{ Carrier Availability Category Responses } & Total \\
\hline Current Week: Apr 29-May 5 & 1 & . & . & . & . & 1 \\
\hline Week 2: May 6-12 & 1 & . & . & . & . & 1 \\
\hline Week 3: May 13-19 & 1 & . & . & . & . & 1 \\
\hline
\end{tabular}

Note: "." denotes no response from carriers.

\begin{tabular}{|l|c|c|}
\hline \multicolumn{3}{|c|}{ Six-Month Snapshot of Average Weekly } \\
Throughput \\
June 2012-November 2012 \\
\hline \multirow{2}{*}{ Container Types } & $\begin{array}{c}\text { Average Total Weekly } \\
\text { Throughput* }\end{array}$ & $\begin{array}{c}\text { Range of Individual } \\
\text { Carrier Responses }\end{array}$ \\
\hline 20ft & 383 & $1 \ldots 300$ \\
40ft & 671 & $15 . . .500$ \\
40ft High Cubes & 797 & $76 \ldots 600$ \\
40ft Refrigerated & 493 & $0 \ldots 203$ \\
20ft Refrigerated & 4 & $0 . . .4$ \\
\hline
\end{tabular}

*Data reflect the sum of the individual carriers' average weekly throughput over a 6-month timeframe 


\section{Understanding this Report}

The weekly Ocean Shipping Container Availability Report (OCSAR) provides a snapshot of container availability for westbound transpacific trade lanes at select intermodal locations around the country. The data presented in this report are gathered from 6 member carriers in the Transpacific Stabilization Agreement (TSA). The TSA carriers include COSCO, Evergreen, Hanjin Shipping, Hapag Lloyd, Yang Ming Transport Corporation, and OOCL.

- The data are estimates of container availability per week by the participating carriers. The carriers determine container availability by comparing current supplies at each of the 18 intermodal locations with the demand for these containers based on up-to-date bookings or reservation information in the westbound transpacific trade lane.

- $\quad$ Each week, participating carriers report the estimated number of containers available for the current week (W1) as well as estimates for the subsequent 2 weeks (W2 and W3).

- Maps on pages 3-5 offer a national overview of weekly container availability, as well as an average of availability over the past 6 months. The weekly national data are derived from the sum of the individual carrier responses at each intermodal location.

- Individual carrier responses are presented by location on pages 6-23 and have been sorted from largest to smallest. To maintain carrier confidentiality, specific carrier identification is not provided.

- Carrier responses showing negative numbers, such as -29 , should be interpreted as meaning that 29 containers are needed by shippers but the carrier is not able to supply them. A positive number, such as 64 , would mean 64 containers are available that are not demanded. An indication of 0 means the carriers have the exact amount of containers needed based on current supply and demand estimates.

- In addition to the weekly container availability data, the participating carriers provide average weekly throughput data for the past 6 months. These data help readers understand the aggregate flow of containers that have moved through the 18 select locations by the 8 participating carriers over the previous 6 months. These data will only be updated every 6 months.

- Availability levels are subject to change, and may be affected by delays in return of equipment to carriers by shippers or consignees, and by free time restrictions imposed by railroads. Due to the dynamic nature of the container industry, carriers cannot be held responsible for the level of availability at the time of inquiry.

- $\quad$ Data presented in the report do not imply availability of chassis.

- Container availability estimates represent availability for all export cargo, not just agricultural products.

- USDA collects these data on a voluntary basis and cannot be held responsible for the status of container availability when a reader makes inquiry with an ocean carrier. USDA relies on the participating ocean carriers to ensure the data is accurate.

- Terminology:

20ft Dry Container (20ft): A standard shipping container that is 20 feet long.

40ft Dry Container (40ft): A standard shipping container that is 40 feet long.

40ft High-Cube (HC): A shipping container which is 40 feet long and 9 feet, 6 inches tall (1 foot taller than a standard $40 \mathrm{ft}$ dry container).

40ft reefer: A refrigerated shipping container which is 40 feet long.

20ft reefer: A refrigerated shipping container which is 20 feet long.

Chassis: A wheeled frame to which a shipping container can be locked for storage or movement. (Plural chassis) 


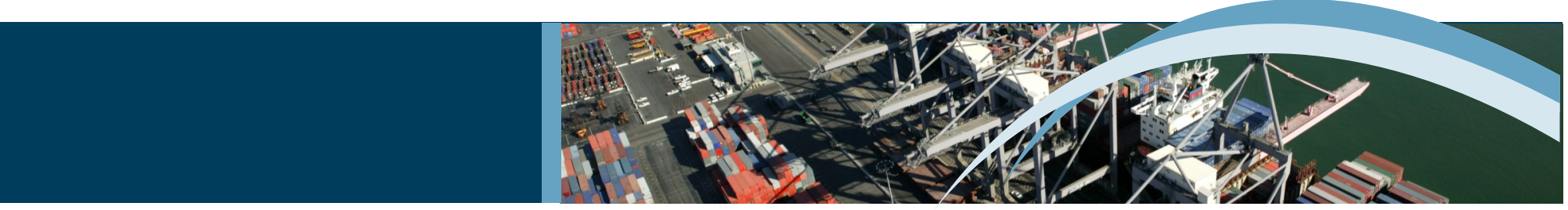

The U.S. Department of Agriculture (USDA) prohibits discrimination against its customers, employees, and applicants for employment on the bases of race, color, national origin, age, disability, sex, gender identity, religion, reprisal, and where applicable, political beliefs, marital status, familial or parental status, sexual orientation, or all or part of an individual's income is derived from any public assistance program, or protected genetic information in employment or in any program or activity conducted or funded by the Department. (Not all prohibited bases will apply to all programs and/or employment activities.)

If you wish to file an employment complaint, you must contact your agency's EEO Counselor (PDF) within 45 days of the date of the alleged discriminatory act, event, or in the case of a personnel action. Additional information can be found online at http:// www.ascr.usda.gov/complaint filing file.html. If you wish to file a Civil Rights program complaint of discrimination, complete the USDA Program Discrimination Complaint Form (PDF), found online at http://www.ascr.usda.gov/complaint filing cust.html, or at any USDA office, or call (866) 632-9992 to request the form. You may also write a letter containing all of the information requested in the form. Send your completed complaint form or letter to us by mail at U.S. Department of Agriculture, Director, Office of Adjudication, 1400 Independence Avenue, S.W., Washington, D.C. 20250-9410, by fax (202) 690-7442 or email at program.intake@usda.gov.

Individuals who are deaf, hard of hearing or have speech disabilities and you wish to file either an EEO or program complaint please contact USDA through the Federal Relay Service at (800) 877-8339 or (800) 845-6136 (in Spanish). Persons with disabilities who wish to file a program complaint, please see information above on how to contact us by mail directly or by email. If you require alternative means of communication for program information (e.g., Braille, large print, audiotape, etc.) please contact USDA's TARGET Center at (202) 720-2600 (voice and TDD).

For any other information not pertaining to civil rights, please refer to the listing of the USDA Agencies and Offices for specific agency information.

Preferred citation:

U.S. Dept. of Agriculture, Agricultural Marketing Service. Ocean Shipping Container Availability Report. April 29, 2015. Web. <http://dx.doi.org/10.9752/TS057.04-29-2015> 